
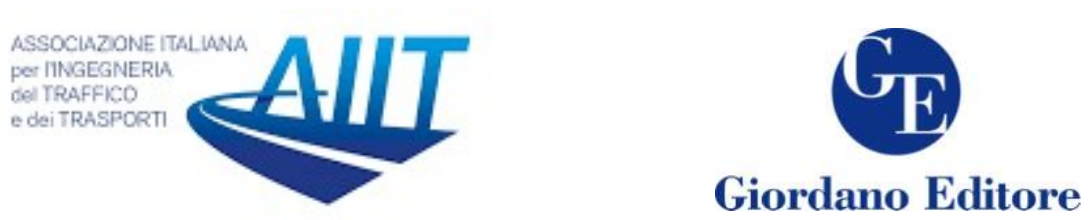

\title{
Giordano Editore
}

\section{The evaluation of the surrogate safety measures along a pedestrian confined ramp of an old bridge}

\section{Chiara Gruden ${ }^{1}$, Tiziana Campisi², Antonino Canale ${ }^{2}$, Matjaž Šraml ${ }^{1}$, Giovanni Tesoriere ${ }^{2}$}

${ }^{1}$ University of Maribor, Faculty of Civil Engineering, Transportation Engineering and Architecture Smetanova 17, 2000 Maribor (Slovenia)

${ }^{2}$ University of Enna Kore,Faculty of Engineering and Architecture - Cittadella Universitaria 94100 Enna

(Italy)

\begin{abstract}
Pedestrians walk daily to meet their basic needs. Therefore, over the years it has been attempted to make urban context more livable, respecting the needs of weak road users. Pedestrian mobility is also growing strongly from the point of view of tourism, especially in the areas where government measures are taken to reduce vehicular flow in order to let the architectural and landscape assets more usable.

Often tourist itineraries are made of narrow areas crossed by numerous pedestrians, leading to possible critical circumstances. The criticality is connected to a likely reduction of safety and use (comfort and possible delays). Confined spaces such as small streets or pedestrian bridges well exemplify the described situation. Particularly, pedestrian bridges are often composed of ramps with variable slopes and consist of different floors, making walking difficult and therefore reducing travel speed and limiting the mobility of users such as handicapped or elderly people.

Starting from the geometrical evaluation of the old bridge of Mostar (BIH), this article provides some ideas for evaluating the safety of pedestrians during the journey on the bridge, under different flow conditions.

The analyses were carried out starting from an estimate of pedestrian flows from video monitoring. Safety was then assessed thanks to the calculation of surrogate measures, based on the trajectories of individuals, obtained through tracking tools.

Camera monitoring and inspections to survey infrastructure's geometric features ease the procedure of data entering into image processing software.

The evaluation of individual pedestrian trajectories and/or their interactions allows to estimate the movement variations through the study of parameters such as speed variation, pedestrian density and surrogate safety factors.

Surrogate safety measures, indeed, are indicators calculated for a chosen pair of users interacting in the detected scene. Among these factors there are: relative speed (Delta-V), Time-To-Collision (TTC), Time Advantage (TAdv) and T2. All the above-mentioned kinds of measures are interesting to study generally the behavioural aspects of road traffic and, specifically, safety level and factors influencing it.

The purpose of this work is to estimate the effects of induced pedestrian mobility on Mostar's Ottoman bridge in terms of safety, with the aim of preventing negative scenarios that could lead to a bad infrastructural level of service.

Keywords: road safety; pedestrian trajectories; surrogate safety measures.
\end{abstract}




\section{Introduction and related works}

Recently mobility is showing a great change in people's habits and way of moving. Sustainable transport modes as walking are spreading in recent years, both regarding daily needs and tourist activities.

Walkable paths are often constituted by narrow facilities, characterized by slopes, steps and various obstacles, which make walking an unsafe task. The increase of walking people is bringing also a grow in pedestrian falls, slipping and collisions with objects, which some researchers have already focused their attention on (R.Methorst, P.Schepers.

N. Christie, M.Dijst, R. Risser, D. Sauter, 2017).

Certainly, this kind of accidents are rare events, thus it is not possible to use traditional accidental data analysis as tool to study them. Moreover, the wide underreporting of this kind of accidental information (Elvik \& Mysen, 1999) makes it not enough reliable for further safety evaluations.

The study of pedestrian trajectories and of their possible encounters can be a solution to the problem of data scarcity: as a matter of fact, these events are much more recurrent than accidents and starting from them, it is possible to work out a surrogate safety evaluation, where "surrogate" indicates a status where no accidents have happened (Laureshyn, Svensson, \& Hydén, 2010).

Many researchers (Schadschneider et al., 2009)(Daamen \& Hoogendoorn, 2003)(Fitzpatrick, Brewer, \& Turner, 2006)(Tanaboriboon, Hwa, \& Chor, 1986)(T. Muraleetharan, T. Adachi, T. Hagiwara, S. Kagaya, 2005)(Laureshyn, Goede, Saunier, \& Fyhri, 2017) have focused their efforts on individuating elements influencing pedestrian safety: the geometry of a section and the more or less dense presence of other walkers in the same are two of the main factors affecting pedestrian behaviour and consequently safety (Schadschneider et al., 2009)(Daamen \& Hoogendoorn, 2003).In addition, gender, age and social aspects act a lot on pedestrian travelling speed and time, modifying their behaviour (Fitzpatrick et al., 2006; Tanaboriboon et al., 1986). Urban areas have been mainly addressed as study locations to highlight how intersection geometry, as well as yielding behaviours and vehicular-pedestrian flow mix can impact on vulnerable road user safety (Laureshyn et al., 2017; T. Muraleetharan, T. Adachi, T. Hagiwara, S. Kagaya, 2005).

Recently, the attention has been turn on surrogate safety indicators: with this term, mainly time and speed measures are defined - like Time-To-Collision, PostEncroachment-Time and relative speed, well representing situations which can lead to possible dangerous conflicts (Laureshyn et al., 2010). These parameters are indeed able to describe and estimate different types of potential accidents, to count them and to provide optimal results to solve daily urban safety problems. In (Elvik \& Mysen, 1999) a complete overview of the surrogate safety theory is introduced, while in (Brijs, n.d.) various techniques applying this theory are reported, as well as useful case studies.

This pro-active approach, with all its advantages - first of all the opportunity of studying safety before an incident happens, has been widely applied to vehicular conflicts, some efforts have been spending on cyclist safety aspects (Laureshyn et al., 2017), but still little works have been developed from pedestrian point of view. Since it is possible to calculate surrogate safety indicators directly from the study of real-world data, and especially from video information, by tracking the road users involved in the scene of interest and by evaluating their trajectories and behavioural parameters (Argoul \& Kabalan, 2017) (Mohamed \& Saunier, 2013), one of the main issues was how to obtain this information, i.e. how to detect pedestrians. This need was a hinder for artificial vision 
research (Enzweiler \& Gavrila, 2009) and traffic micro-simulation (Tesoriere, Campisi, Canale, \& Zgrablić, 2018) when referring to pedestrians and cyclists. As a matter of fact, pedestrian detection shows much more difficulties than vehicular one: its target are people, who often have different and changeable features, assuming various shapes and moving in surrounding environments, which also vary frequently (Lin, Gang Hua, \& Davis, 2010). Moreover, pedestrian body sizes differ a lot from one individual to the other and also their height is extremely various: particularly this last fact induces many issues in pedestrian detection, as various magnitudes in imaging techniques are obtained by calculating the distance difference between the selected individual and camera's coordinates.

The first research work in this area focused on the prediction of pedestrian trajectories through the comparison of many data or through the topological characteristics of the places present in a map (T. Ikeda, Y. Chigodo, D. Rea, F. Zanlungo, M. Shiomi, 2013).

The main disadvantage of these methods is their dependence on specific background information and their limitation in prediction time and length. A two-level forecasting process has also been proposed (Foka \& Trahanias, 2010).

It defines a short-term prediction based on polynomial neural networks, and a longterm probability-based forecast of transition between a series of manually defined critical points. This technique, however, provides only the future position of the detected pedestrian and does not take into account the problems of time or speed. A more recent approach proposes a probabilistic method to determine pedestrian trajectory (Tamura, Terada, Yamashita, \& Asama, 2013).

This methodology classifies the behaviors of pedestrians in defined schemes, subjected to observation. This technique, however, does not consider the environment, providing only simple trajectories based on the direction of movement. Some studies have been addressed considering the definition of pedestrian trajectories through video acquisition and processing with dedicated tracking tools (Gorrini, Vizzari, \& Bandini, 2016).

The development of improved detecting and tracking techniques also suitable for pedestrian and cyclist analysis, has allowed to apply the calculation of surrogate safety measures to these road users, too. In (Laureshyn et al., 2010) the first concept related to this kind of studies are given, while in (Laureshyn et al., 2017)three safety techniques have been compared, calculating surrogate safety measures for cyclists and relying on real-world data extracted thanks to a semi-automatic detecting and tracking tool. (Chen, Zeng, Yu, \& Wang, 2017) used aerial images and a detecting and tracking tool to elaborate a surrogate safety analysis of pedestrians and right turning vehicle conflicts at a non-signalized crosswalk on a 4-leg intersection.

Within this framework it stands this research: the authors focus on pedestrian flows and trajectories, elaborated from video-recordings using a semi-automated detection and tracking tool still not extensively applied to pedestrian studies. Video footage elaboration allowed to collect interesting trajectory data which permit the further estimation and analysis of surrogate safety measures referred to pedestrians.

Aim of the work is to study pedestrian behaviour and safety by evaluating walkers' trajectories and conflicts in a confined, narrow space, totally dedicated to pedestrian movement, where there are no external road-related factors influencing people. In this way it will give an insight of pedestrian undisturbed behaviour, pointing out normally underestimated, walking risky situations, i.e. pedestrian collisions. 

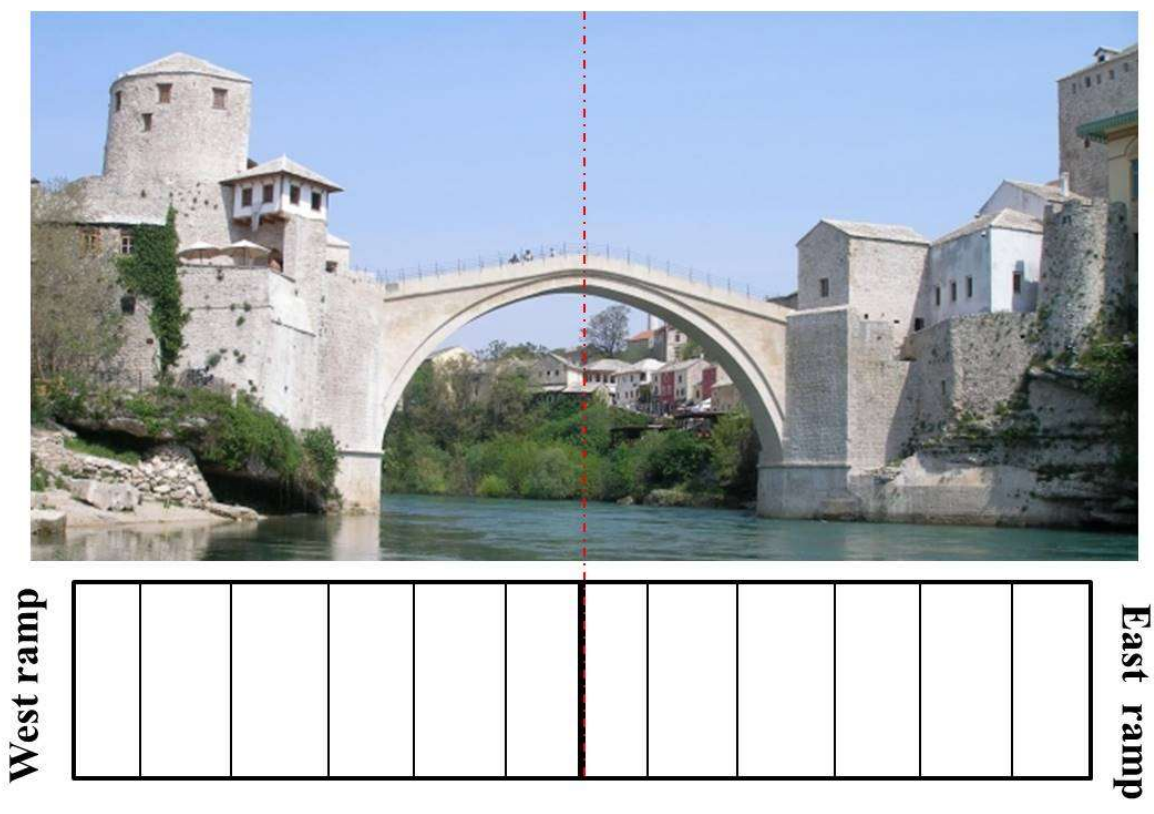

Figure 1: The monitored area of the old bridge (Stari Most) of Mostar city (BIH).

\section{Methodology}

Aim of the research is to develop a study of the natural behaviour of pedestrians in a totally dedicated and confined facility, characterized by high pedestrian volumes. Such a location would allow not only to deep analyze pedestrian movements, but also to focus on safety issues, which still have not been extensively treated, looking at walkers' real, undisturbed behaviour.

\subsection{Case study location}

To reach the recalled goal, the location chosen is Mostar (BIH) and specifically, the old bridge of the city, named Stari Most. This place has been selected because of its particular features, both in environment and traffic.

As regarding the environmental aspects, on the one hand, it is a narrow and long area, similar to many other common facilities, like sidewalks and corridors; on the other hand, it has some structural peculiarities, which could affect walkers' behaviour. As a matter of fact, the facility is characterized by two ramps, one ascending and one descending, each one with a constant slope of $20 \%$. Each ramp has a width of 4,0 m and a length of 9,0 m. Along the bridge there are some particularly shaped steps: these can be defined as kerbs, created in order to block the slipping feet of walking people. Kerbs have an extent equal to the width of the bridge, i.e. $4,0 \mathrm{~m}$, and they are $0.05 \mathrm{~m}$ wide. Between two consecutive kerbs an area with sizes of 4,0x0,30 $\mathrm{m}$ is comprised.

Focusing on traffic features, pedestrian flow is very high, it is bi-directional and mixed, composed of both tourists and locals. Because of bridge's surroundings, many tourists make a lot of stops and delays, in order to observe the panorama. On the other side locals try to exit the zone as faster as possible. 
Based on this information, Stari Most seemed an ideal location for the aim of this research, because its global features let the developed method be easily repeated over many similar infrastructures, e.g. corridors, pedestrian bridges, sidewalks, etc.; at the same time, its peculiarities, like the particularly shaped steps and the ramp slope, can affect people's behaviour.

\subsection{Used method}

The method used to develop the research is based on four main steps: the geometrical and functional analysis of the location under study, the development of on-site video surveys, the extraction of traffic and safety data from the videos via a semi-automatic detection and tracking tool and the analysis of the obtained information (Fig.1). This general procedure permits to obtain data useful both for a global assessment of pedestrian dynamics and infrastructural level of service, and for an estimation of the facility level of safety.
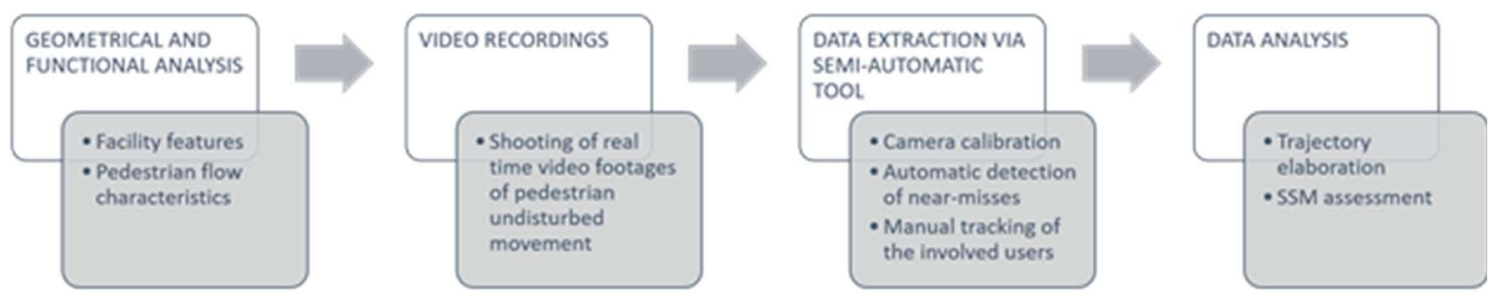

Figure 2: 4-step procedure followed to develop the assessment.

The geometrical and functional analysis allowed to achieve data about the environment where pedestrians walks and to stress out elements, which can cause particular dynamics. This step has been developed matching together mapping information, Autocad drawings and visual observations. Referring to the case study, the facility is characterized by two $9,0 \mathrm{~m}$ long ramps of opposite constant slope - $20 \%$, and each one made up of $300,05 \mathrm{~m}$ wide kerbs. The whole bridge has a width of $4,0 \mathrm{~m}$ and it constitutes in its final sections a bottleneck to the oncoming flows.

The second step consisted in recording videos of the natural behaviour of people, without disturbing them or letting them notice the presence of the cameras. Video cameras XXYYY have been placed in strategic locations, which permitted on a side to have a whole view of the examined facility and to shoot in real-time people using the bridge, and on the other side to be hide from pedestrians view. Recordings have been taken in September 2018, period when both the presence of tourists and locals is high, during the peak hour.

The recorded footages have been elaborated during the third step, through a semiautomatic tool for pedestrian detection and tracking. The utilized device allows to detect interesting situations, and to track each involved pedestrian. In this way it is possible to obtain trajectory data, as well as speeds and accelerations for the tracked users in both directions and-consequently- to highlight, count and deeper analyse near-misses and the areas where they happened the most. 
Starting from video recordings, four important tasks have to be followed to obtain useful data. First and foremost, it is essential to run camera calibration, linking the shot frames to an orto-photo of the interested area: this step allows to create a coordinate system, which it will be referred to during the whole video elaboration. In the examined case, the absolute error values on map and camera views equal respectively $12 \mathrm{~cm}$ and 4 pxl, so very limited outliers. The second passage is to identify the interesting events, i.e. near-accidents. Once pinpointed them, it is possible to automatically save shorter footages - $14 \mathrm{~s}$ overall, including the selected scene. The obtained videos are the detections, on which the tracking step will be worked out. The tracking phase consists in characterizing each road user with a "shaped box". This box has to be manually located around the user at each selected frame-step in order to obtain its trajectory. Once the manual tracking is concluded, the trajectory can be smoothed to achieve a continuous one, and outputs can be provided. Among all the outputs, two groups of magnitudes can be distinguished: behavioural magnitudes, provided via .csv files, and surrogate safety measures, which can be easily copied and pasted from the tool's graphical user interface to Excel. Behavioural magnitudes consist in the definition for each tracked pedestrian of its position, speed, acceleration (and other more specific values) at each frame.

Surrogate safety measures, instead, are indicators calculated for a chosen pair of users interacting in the detected scene. Both kind of measures are interesting to study generally the behavioural aspects of road traffic and specifically safety level and factors influencing it. Of course, for a more detailed description of the program use, it is highly recommended to visit the webpage of InDeV project ("InDeV - Home," n.d.). Among all the existing surrogate safety measures, T-Analyst provides the automatic calculation of the parameters described in Table 1.

\section{Results and discussion}

During the analysis of the recorded video footages, various flow conditions have been observed and specifically four of them have been selected as scenarios to be examined.

Four scenarios - see Fig. 2- have been chosen in order to study the dynamics of a labelled walker, defined Beta-tester, with homogeneous characteristics, under different flow conditions - from low to high density situations.

The Beta-tester has been selected on the basis of its age and gender, in order to represent the majority of people walking on the bridge. Specifically, a middle aged (40-50 years old) man has been selected as labelled person.

The defined scenarios can be described as follows:

SCENARIO 1: two individuals facing each other, walking in opposite directions. The two persons are almost isolated and the main interaction happens only between them.

SCENARIO 2: a total of four people is tracked, two for each flow direction. Also in this case, the main interactions happen among the considered pedestrians, even if other people are present in the detected scene.

SCENARIO 3: three people walking in each direction, for a total amount of six individuals. The scene is getting crowder as the previous one and the effects of other pedestrians increase. 
SCENARIO 4: four pedestrians face other four pedestrians coming from the opposite side. Totally eight pedestrians are tracked. The tracking phase becomes tricky, because of the crowd present in the scene. The interactions among all people in the scene become high.

SCENARIO $1-1: 1$

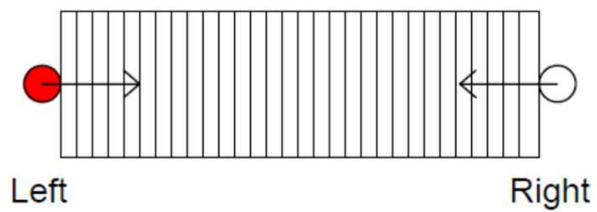

SCENARIO 3 - 3:3

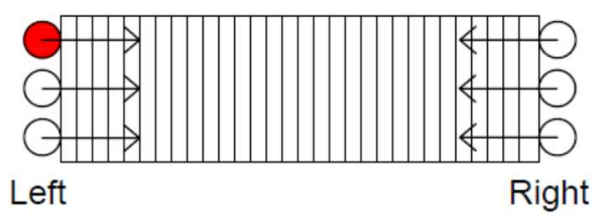

SCENARIO 2 - 2:2

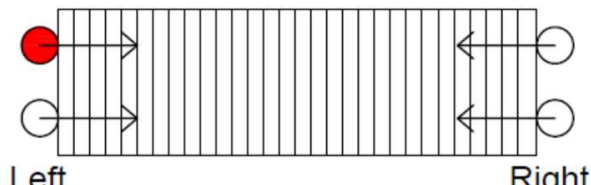

Right

Figure 3: The 4 studied scenarios. The red dot represents the Beta-Tester.

A maximum of four pedestrians per flow direction has been tracked, because it resulted the maximum number of people able to pass in the considered section without overlapping, thus creating high density conditions, but still not emergency ones. This feature allowed also a reliable (though tricky) tracking phase. From video footages firstly flow percentage have been recorded. Specifically, the total flow and the flow in each direction have been calculated, counting people passing on the 18th step of the ramp. Flux has been counted on a $2 \mathrm{~s}$ basis and then transformed in a $1 \mathrm{~s}$ and 1 min-based flow. Table 1 reports the flow percentage for the different scenarios. Meanly, the total flow was 40 peds/min, with a peak of 64 peds/min in the third scenario.

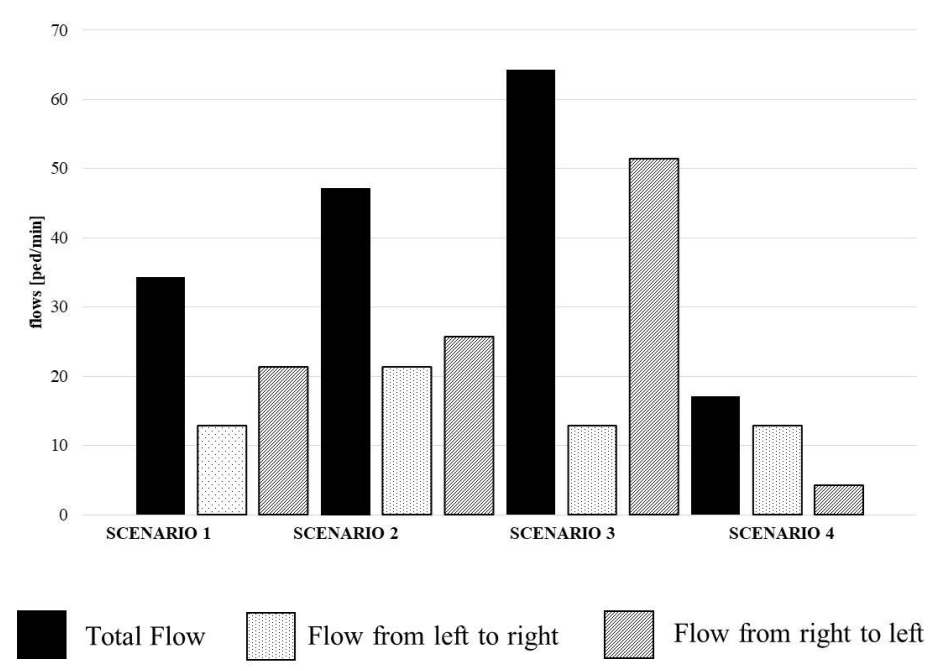

Figure 4: Pedestrian flow distribution considering different scenarios. 
Already from these data it is noticeable how in low density situations, i.e. scenarios 1 and 2 , flows are mostly equal in both directions, while in high density conditions there is one dominating direction. The tracking phase permitted to obtain the trajectory of each pedestrian walking during the detected scene. In Figure 3 the trajectories of walkers divided per scenario are displayed. The continuous line represents the Beta-tester, while the others are the trajectories of the remaining people. Since the graphs plot the $\mathrm{x}$ and $\mathrm{y}$ coordinates of the tracked pedestrians, time variable is not considered in the figure. This means that trajectories which overlap do not correspond to overlapping people - since this condition has been avoided. As regarding the coordinate system: the x-axis was set in correspondence of the length of the ramp, while $y$-axis corresponds to bridge width.

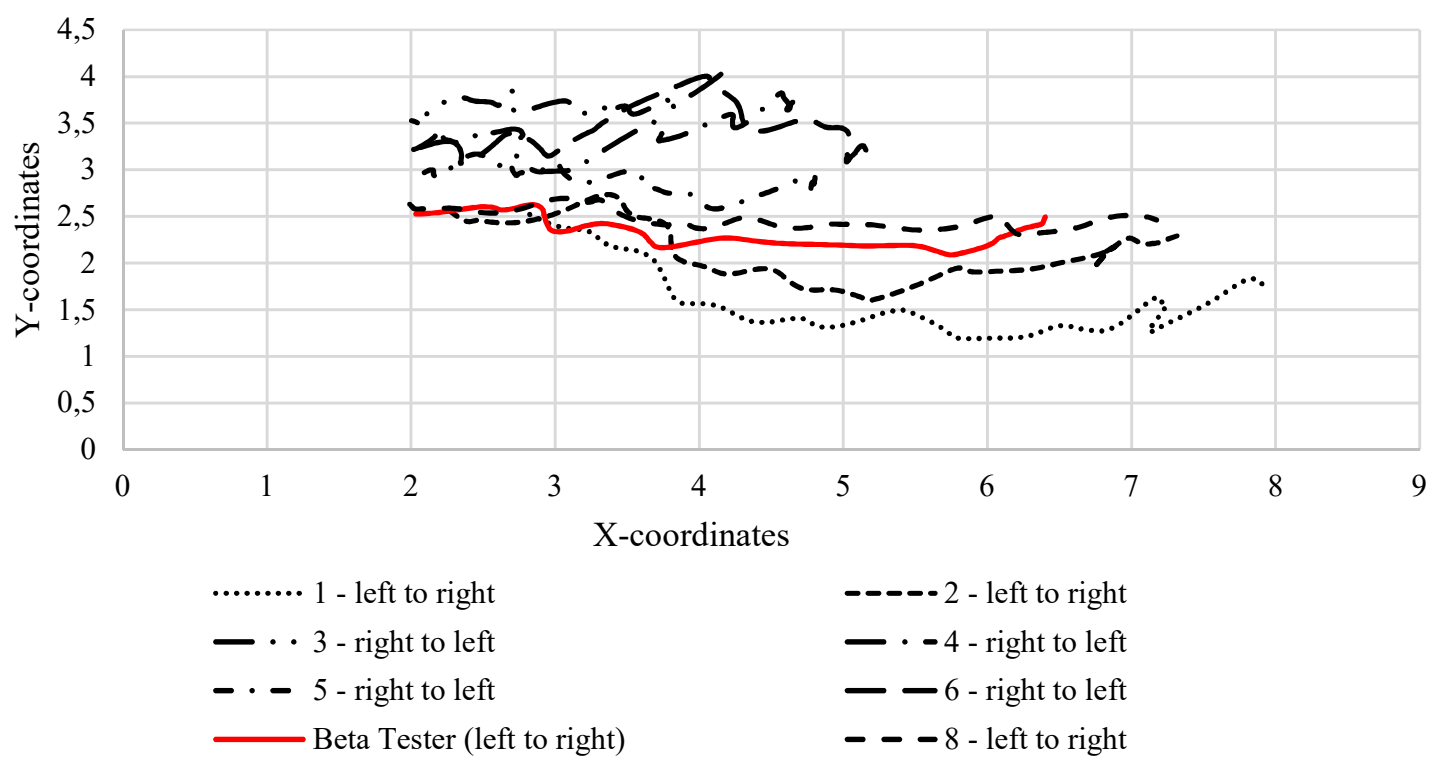

Figure 5: Trajectories of beta tester considering selected scenarios.

Analogous graphs have been obtained for the other three scenarios and from their comparison the following can be stated: flow conditions are increasing by increasing scenario, with four people walking in scenario 1, six walkers in scenarios 2 and 3, and eight in scenario 4. It is also noticeable the difference in the influence of all pedestrians on the considered ones: in the first two graphs people are comfortably walking without affecting very much one the other, while in the last two the interactions become much stronger and also bottleneck effects are visible. Looking at Beta-tester's trajectory, it is appreciable the will of the considered walker to move as much as possible on the longitudinal axis of the bridge, confirming the general willingness of pedestrians to keep the higher distance possible from obstacles and walls. Also, pedestrian trajectories become much more homogeneous as the number of walker grows.

After the analysis of the whole scene, considering each pedestrian walking on the bridge, only people involved in near-misses were studied. This sort of restricted analysis allowed to achieve speed data and particularly, relative speed between two involved walkers. During the speed data analysis, it has been noticed that relative speed, Vrel, was not reported at some points, though velocities of each individual were available. The reason of these gaps stands in the vectorial definition of the indicator: relative speed, 
indeed, is calculated - as quoted from (Lili Pu and Rahul Joshi, 2008) - like "the difference in vehicle velocities (or trajectories), such that if v1 and v2 are the velocity vectors of the first and second vehicles respectively, then DeltaS $=\|$ v1 - v2 $\| "$. So, if two trajectories are parallel, the value of relative speed will be null. In these cases, the achieved data show a gap, and consequently, surrogate safety measures do it too, since the users are not walking on a possible conflict line.

Taking into account the reliable speed data, a mean value of $0,81 \mathrm{~m} / \mathrm{s}$ with a standard deviation of $0,13 \mathrm{~m} / \mathrm{s}$ between two facing pedestrians has been achieved.

From near-misses analysis, also TAdv, T2 and TTC have been provided. In this research the trend of TAdv over time, the values of T2 in relation to relative speed and the null points of TTC in function of space have been studied.

In Fig. 4, T2 in relation to Vrel is plotted. In the recalled graph, the values of T2 and Vrel of the near-misses ivolving the Beta-tester in all four scenarios are displayed. It can be seen that the majority of the data are comprised in the range $[0,4 ; 1,0] \mathrm{m} / \mathrm{s}$ as regarding relative speed and they belong to the range $[0 ; 12]$ s considering $\mathrm{T} 2$.

The comparison of the trend of TAdv over the four scenarios (Figure 5) is a little bit trickier than the previous one, since the frames, which near-misses start at, differ from case to case.

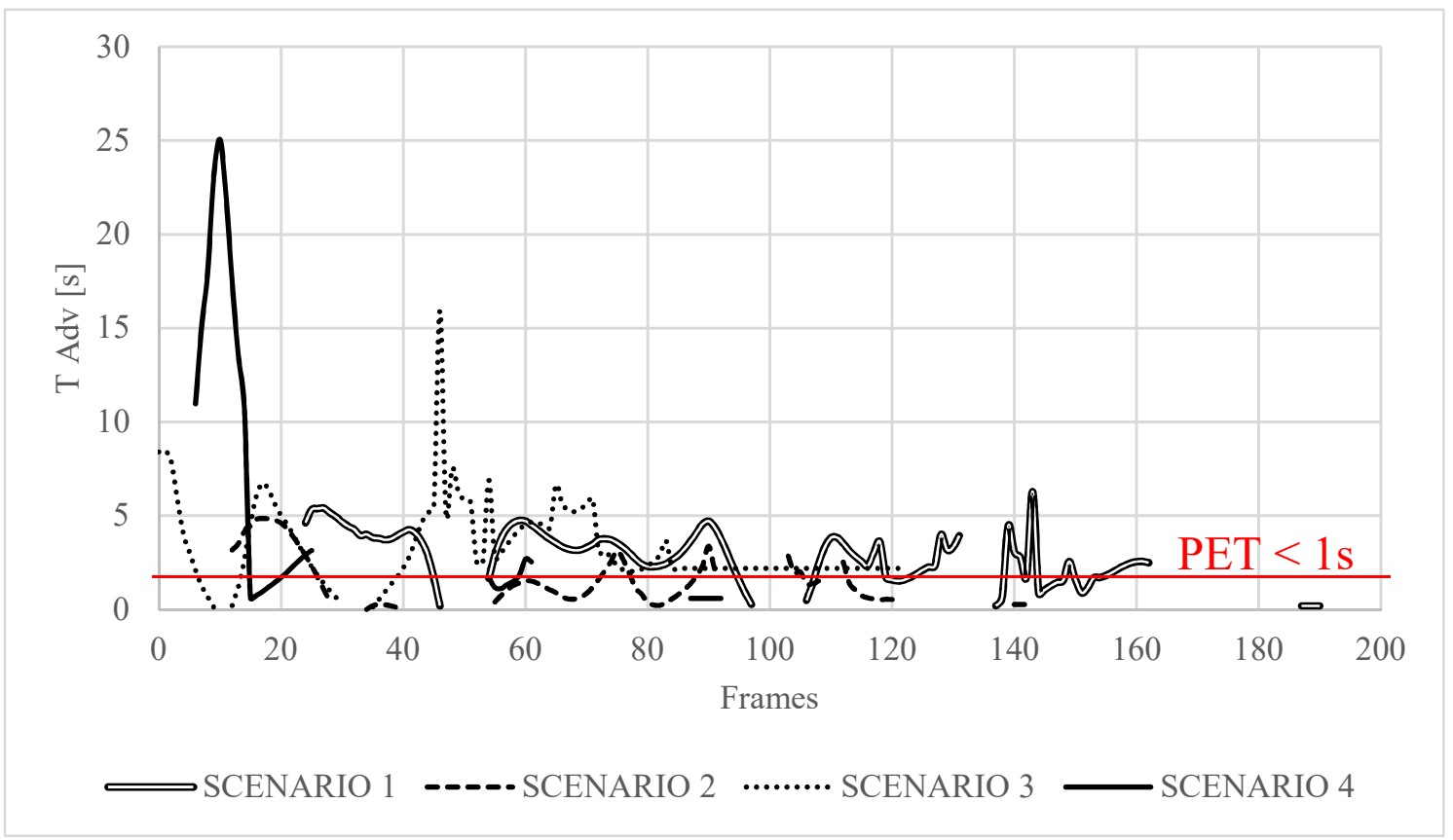

Figure 6: TAdv and PET distribution considering the monitored scenarios.

Generally, it can be said that TAdv shows a peak at the beginning of the encounters, and decreases till reaching a quite constant value by increasing time. The range, which the most of TAdv values belong to, is [0;5] s, halving T2 range.

Finally, an evaluation of TTC in relation to space was made. Specifically, aim of this estimation is to pinpoint the locations of the bridge, where TTC equals 0 . This condition represents, indeed, the points where conflicts happen. Focusing on this condition, the following can be stated: conflict points tend to set where there are higher density situations and in relationship to the amount of flow in the two directions. If the two flows are similar, the encounters happen in the middle of the ramp, if one flow is higher than 
the other, the conflicts move to one of the ramp ends. Fig. 6 (left) recalls the locations where encounters happen, showing the bridge ramp divided in six zones, each containing five kerbs. These areas have been depicted with a colour map on the basis of the number of times $\mathrm{TTC}=0$. The worst situation is coloured in red, and it indicates an area where more than 10 encounters happened. The risk level decreases by moving from red to green areas, where no near-misses have been detected.

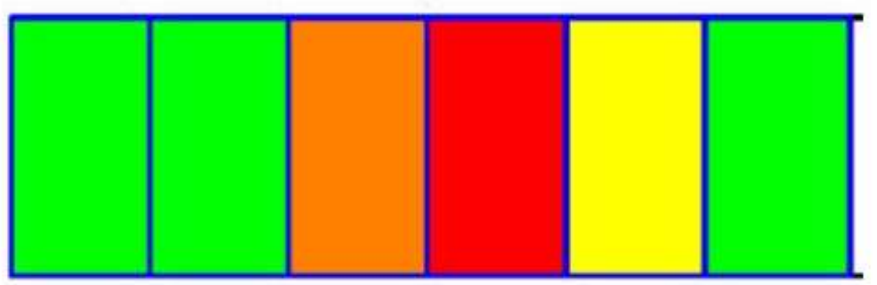

zone 1 zone 2 zone 3 zone 4 zone 5 zone 6

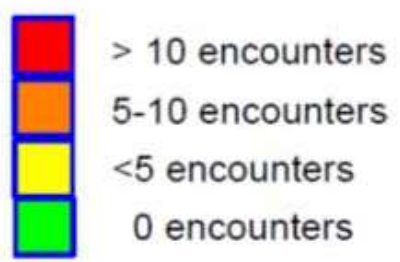

Figure 7: SSM values distribution.

Taking into account all the elaborated detections, the most critical zone of the ramp is the central one, where most of the observed pedestrian conflicts happened. From the previous observations, it is also clear that the preferred path on the ramp is the one on the axis of the facility and the most critical zone is the ramped center. These facts lead to the statement that, in a confined space, characterized by a bi-directional pedestrian flow, a solution to reduce impacts among opposing pedestrians could be the positioning on the axis of the ramp central part of a dividing element, such as a short wall or a handrail. Such a conclusion has been drawn also by (Lili Pu and Rahul Joshi, 2008) by analyzing controlled experiments through bottlenecks and by (von Krüchten, Müller, Svachiy, Wohak, \& Schadschneider, 2016) studying emergency situations. The examined case does not belong to those fields, but still, it confirms their results also regarding normal flow conditions.

\section{Conclusions}

The developed research shows the importance of using real-world trajectory data to study pedestrian behaviour and safety, by the use of surrogate safety method.

Among the numerous advantages shown by the method, the most noticeable is that it is not necessary to wait for the incident to happen, in order to study pedestrian safety.

The surveyed location, dedicated only to pedestrian use and characterized by high and mixed walking volumes, is an environment characterized by very specific features: the space is narrow and it presents its own difficulties, i.e. the slope and the stairs, but at the same time it is a easily replicable place, making it possible to apply the obtained results 
in many other similar spaces, like pedestrian bridges, corridors, physically separated walkways, etc....

One of the aims was to extend the application of a semi-automatic tool to detect and track road users, also to pedestrian movement. The developed case shows that it is possible to apply it to pedestrian study and that it allows also the calculation of surrogate safety indicators at the basis of the recalled method.

Various types of data have been achieved and elaborated: behavioural data and, specifically, pedestrian trajectories both for single encounters and for the whole considered scenario, surrogate safety measures and particularly T2, TAdv and TTC, that allowed to stress the most dangerous areas in the ramp. The study of T2 - Vrel relationship highlighted the range of the two magnitudes, specifically $[0,4 ; 1,0] \mathrm{m} / \mathrm{s}$ for Vrel and $[0 ; 12] \mathrm{s}$ for T2. As regarding TAdv, the threshold given in (Laureshyn et al., 2017 ) to Pet to consider an encroachment risky is 1s. Looking at Fig.5, it is noticeable that there are various values under the threshold, confirming the near-misses found analyzing TTC. To understand where are placed the most redundant conflict points, the ramp has been divided in six zones, each one composed of five steps, and the number of points where TTC equals zero have been counted. This analysis led to the conclusion that not the whole ramp is subjected to the same risk probability, but its middle zones are the most dangerous.

The work has highlighted that the majority of pedestrians has a similar behaviour, moving as straight as possible along the ramp and trying to walk on the axis of the same, avoiding other walkers coming in the opposite direction. From this consideration it follows also that on the axis is set the majority of the encounters.

So both the longitudinal and lateral axis of the ramp represent the most critical areas of the location, and the ones where it can be useful to put some safety devices.

Finally, this research sets the basis for a further and deeper analysis, where trajectory data linked to surrogate parameters would lead to a global assessment of pedestrian safety. From this viewpoint, next steps will be the simulation of the same environment and the related estimate of surrogate safety measures deriving from the simulated model, and the simulation of a modification of the environment, e.g. putting some longitudinal splitting elements in the middle of the ramp, to assess if it can effectively lead to an improvement of safety.

\section{References}

Argoul, P., \& Kabalan, B. (2017). Pedestrian Trajectories and Collisions in Crowd Motion. https://doi.org/10.1007/978-3-662-52696-5_6

Brijs, T. (n.d.). How to analyse accident causation? A handbook with focus on vulnerable road users Evelien Polders \&.

Chen, P., Zeng, W., Yu, G., \& Wang, Y. (2017). Surrogate Safety Analysis of PedestrianVehicle Conflict at Intersections Using Unmanned Aerial Vehicle Videos. Journal of Advanced Transportation, 2017. https://doi.org/10.1155/2017/5202150

Daamen, W., \& Hoogendoorn, S. P. (2003). Experimental Research of Pedestrian Walking Behavior. Transportation Research Record: Journal of the Transportation Research Board, 1828(1), 20-30. https://doi.org/10.3141/1828-03

Elvik, R., \& Mysen, A. (1999). Incomplete Accident Reporting: Meta-Analysis of Studies Made in 13 Countries. Transportation Research Record: Journal of the 
Transportation Research Board, 1665(1), 133-140. https://doi.org/10.3141/1665-18 Enzweiler, M., \& Gavrila, D. M. (2009). Monocular pedestrian detection: Survey and experiments. IEEE Transactions on Pattern Analysis and Machine Intelligence, 31(12), 2179-2195. https://doi.org/10.1109/TPAMI.2008.260

Fitzpatrick, K., Brewer, M. A., \& Turner, S. (2006). Another Look at Pedestrian Walking Speed. Transportation Research Record: Journal of the Transportation Research Board, 1982(1), 21-29. https://doi.org/10.1177/0361198106198200104

Foka, A. F., \& Trahanias, P. E. (2010). Probabilistic Autonomous Robot Navigation in Dynamic Environments with Human Motion Prediction. International Journal of Social Robotics, 2(1), 79-94. https://doi.org/10.1007/s12369-009-0037-z

Gorrini, A., Vizzari, G., \& Bandini, S. (2016). Towards Modelling Pedestrian-Vehicle Interactions: Empirical Study on Urban Unsignalized Intersection. Retrieved from http://arxiv.org/abs/1610.07892

InDeV - Home. (n.d.). Retrieved November 27, 2019, from https://www.indevproject.eu/InDeV/EN/Home/home_node.html

Laureshyn, A., Goede, M. de, Saunier, N. , \& Fyhri, A. (2017). Cross-comparison of three surrogate safety methods to diagnose cyclist safety problems at intersections in Norway. Accident Analysis and Prevention, 105, 11-20. https://doi.org/10.1016/j.aap.2016.04.035

Laureshyn, A., Svensson, Å., \& Hydén, C. (2010). Evaluation of traffic safety, based on micro-level behavioural data: Theoretical framework and first implementation. Accident Analysis and Prevention, 42(6), 1637-1646. https://doi.org/10.1016/j.aap.2010.03.021

Lili $\mathrm{Pu}$ and Rahul Joshi. (2008). Surrogate Safety Assessment Model (SSAM)SOFTWARE USER MANUAL.

Lin, Z., Gang Hua, \& Davis, L. S. (2010, March 1). Multiple instance fFeature for robust part-based object detection. 405-412. https://doi.org/10.1109/cvpr.2009.5206858

Mohamed, M. G., \& Saunier, N. (2013). Motion Prediction Methods for Surrogate Safety Analysis. Transportation Research Record: Journal of the Transportation Research Board, 2386(1), 168-178. https://doi.org/10.3141/2386-19

R.Methorst, P.Schepers. N. Christie, M.Dijst, R. Risser, D. Sauter, B. van W. (2017). 'Pedestrian falls' as necessary addition to the current definition of 2 traffic crashes for improved public health policies. Journal of Transport \& Health, 6, 10-12. Retrieved from https://discovery.ucl.ac.uk/id/eprint/1544675/1/Christie_pedestrian falls_improved public health policies_.pdf

Schadschn̄eider, A., Klingsch, W., Klüpfel, H., Kretz, T., Rogsch, C., \& Seyfried, A. (2009). Evacuation Dynamics: Empirical Results, Modeling and Applications. In Encyclopedia of Complexity and Systems Science (pp. 3142-3176). https://doi.org/10.1007/978-0-387-30440-3_187

T. Ikeda, Y. Chigodo, D. Rea, F. Zanlungo, M. Shiomi, T. K. (2013). Modeling and prediction of pedestrian behavior based on the sub-goal concept. Robotics, 10. Retrieved from https://books.google.hr/books?hl=it\&lr=\&id=aSodAAAAQBAJ\&oi=fnd\&pg=PA1 $37 \& d q=I k e d a,+T .,+$ Chigodo, + Y., + Rea, + D., + Zanlungo, + F., + Shiomi, + M., $+\% 26+\mathrm{K}$ anda, + T. $+(2013) .+$ Modeling + and + prediction + of + pedestrian + behavior + based + on + the + sub-goal + concept\&ots $=$ NvzbzYEhaR\&sig $=9 \mathrm{Nb}$

T. Muraleetharan, T. Adachi, T. Hagiwara, S. Kagaya. (2005). METHOD TO DETERMINE PEDESTRIAN LEVEL-OF-SERVICE FOR CROSSWALKS AT 
URBAN INTERSECTIONS. Journal of the Eastern Asia Society for Transportation Studies, 6, 127-136. https://doi.org/10.11175/easts.6.127

Tamura, Y., Terada, Y., Yamashita, A., \& Asama, H. (2013). Modelling Behaviour Patterns of Pedestrians for Mobile Robot Trajectory Generation. International Journal of Advanced Robotic Systems, 10(8), 310. https://doi.org/10.5772/56668

Tanaboriboon, Y., Hwa, S. S., \& Chor, C. H. (1986). Pedestrian Characteristics Study in Singapore. Journal of Transportation Engineering, 112(3), 229-235. https://doi.org/10.1061/(ASCE)0733-947X(1986)112:3(229)

Tesoriere, G., Campisi, T., Canale, A., \& Zgrablić, T. (2018). The Surrogate Safety Appraisal of the Unconventional Elliptical and Turbo Roundabouts. Journal of Advanced Transportation, 2018. https://doi.org/10.1155/2018/2952074

von Krüchten, C., Müller, F., Svachiy, A., Wohak, O., \& Schadschneider, A. (2016). Empirical Study of the Influence of Social Groups in Evacuation Scenarios. In Traffic and Granular Flow '15 (pp. 65-72). https://doi.org/10.1007/978-3-31933482-0_9 